\title{
Barreiras à implementação de recomendações para assistência ao parto normal: revisão rápida de evidências
}

\author{
Cintia de Freitas Oliveira, ${ }^{1}$ Aline Ângela Victoria Ribeiro, ${ }^{1}$ Cézar D. Luquine Jr., ${ }^{1}$ Maritsa Carla de \\ Bortoli, ${ }^{1}$ Tereza Setsuko Toma, ${ }^{1}$ Evelina Maria Gracia Chapman ${ }^{2}$ e Jorge Otávio Maia Barreto ${ }^{2}$
}

Como citar Oliveira CF, Ribeiro AAV, Luquine Jr. CD, de Bortoli MC, Toma TS, Chapman EMG, et al. Barreiras à implementação de recomendações para assistência ao parto normal: revisão rápida de evidências. Rev Panam Salud Publica. 2020;44:e132. https:// doi.org/10.26633/RPSP.2020.132

RESUMO

Objetivo. Identificar potenciais barreiras à implementação das recomendações das Diretrizes Nacionais de Assistência ao Parto Normal a partir das melhores evidências globais disponíveis.

Métodos. Realizou-se uma revisão rápida com consulta a seis bases de dados em março/abril de 2019. Foram selecionados estudos secundários publicados em inglês, espanhol ou português sobre barreiras de qualquer natureza que pudessem ser relacionadas à implementação das recomendações das Diretrizes.

Resultados. Foram incluídos 23 documentos (21 revisões sistemáticas e dois guias de prática clínica). As barreiras identificadas foram agrupadas em 52 categorias por semelhança de significado e, em seguida, reorganizadas em nove núcleos temáticos: modelo de atenção ao parto e nascimento, gestão de recursos humanos, crenças e saberes, relações de gênero, gestão de serviços de saúde, atitudes e comportamentos, comunicação, condições socioeconômicas e interesses políticos.

Conclusões. Os resultados mostraram que a implementação das Diretrizes pode requerer abordagens combinadas para o enfrentamento de diferentes barreiras. O engajamento de gestores e profissionais de saúde no processo de mudança do modelo de atenção ao parto e nascimento e o envolvimento de usuários são indispensáveis para o sucesso da implementação. São necessárias, ainda, ações intersetoriais para melhorar as condições socioeconômicas de mulheres e famílias e para combater as iniquidades de gênero.

Palavras-chave Políticas informadas por evidências; ciência da implementação; guias de prática clínica como assunto; parto; Brasil

Nas últimas décadas, houve avanços importantes no atendimento à gestante, à parturiente e ao recém-nascido no Brasil, mas persistem desafios relacionados à qualidade da atenção ao parto (1). O aspecto mais visível do desafio a ser enfrentado são as taxas de cirurgia cesariana, que aumentaram de 15\% em 1970 para 56\% em 2015, apesar de várias medidas políticas adotadas nos últimos 20 anos (1-3). Exemplos de iniciativas recentes pelo Ministério da Saúde incluem a Rede Cegonha, de 2012; e os projetos Parto Adequado, de 2015, e Aprimoramento e Inovação no Cuidado e Ensino em Obstetrícia e Neonatologia (ApiceOn), de 2017.

\footnotetext{
1 Secretaria de Estado da Saúde de São Paulo, Instituto de Saúde, São Paulo (SP), Brasil.
}

Além dessas iniciativas, em 2017 foram elaboradas as Diretrizes Nacionais de Assistência ao Parto Normal $(1,4)$. As Diretrizes contêm 225 recomendações distribuídas em oito seções: 1) local de assistência ao parto, 2) cuidados gerais durante o trabalho de parto, 3) alívio da dor no trabalho de parto, 4) assistência no primeiro período do parto, 5) assistência no segundo período do parto, 6) assistência no terceiro período do parto, 7) cuidados maternos imediatamente após o parto e 8) assistência ao recém-nascido (4).

Embora sejam fundamentais para garantir a qualidade na assistência à saúde, documentos como diretrizes ou guias de

\footnotetext{
2 Fundação Oswaldo Cruz (Fiocruz), Brasília (DF), Brasil. $\bowtie$ Jorge Otávio Maia Barreto, jorge.barreto@fiocruz.br
} 
prática clínica podem ser subutilizados (5) caso seu processo de implementação seja mal planejado ou ineficiente (6). Nesse planejamento, é importante a caracterização de potenciais barreiras que representem desafios aos diversos atores envolvidos nos vários níveis de organização nos sistemas de saúde (7). Sendo assim, o objetivo do presente estudo foi revisar as melhores evidências globais disponíveis para identificar barreiras à implementação das recomendações das Diretrizes Nacionais de Assistência ao Parto Normal no Brasil.

\section{MATERIAIS E MÉTODOS}

A presente revisão sistemática integra um projeto de pesquisa da iniciativa "Incorporação de pesquisas para os Objetivos de Desenvolvimento Sustentável", liderada pela Organização Pan-Americana da Saúde (OPAS), e é apresentado juntamente com um segundo artigo nesta mesma edição temática, denominado "Barreiras e estratégias para implementação de Diretrizes Nacionais do Parto Normal no Brasil". Realizou-se uma revisão sistemática rápida utilizando atalhos metodológicos para produzir uma síntese das melhores evidências disponíveis no tempo oportuno para atender a demanda específica de uma política pública de saúde (8). O protocolo desta revisão está disponível no Material Suplementar (página 1).

Foram incluídos apenas estudos secundários (revisões sistemáticas, sínteses de evidências qualitativas, mapeamentos sistemáticos, revisões narrativas e guias de prática clínica), publicados em português, inglês ou espanhol, que abordaram barreiras à implementação de uma ou mais recomendações das Diretrizes.

\section{Buscas}

As buscas foram realizadas de 21 de março a $1^{\circ}$ de abril de 2019 em seis fontes de dados, sem limitação de data de publicação. Na base PubMed (https: / / pubmed.ncbi.nlm.nih. gov/), utilizaram-se os termos MeSH "parturition" (entry terms "Parturitions", "Birth", "Births", "Childbirth", "Childbirths") e "guideline adherence" (entry terms "Adherence, Guideline", "Policy Compliance", "Compliance, Policy", "Protocol Compliance", "Compliance, Protocol", "Institutional Adherence", "Adherence, Institutional"). No Portal Regional da Biblioteca Virtual em Saúde (BVS) (https: / /bvsalud.org/), os Descritores em Ciências da Saúde (DeCS) em português foram "parto normal" e "parto humanizado", restringindo a busca a humanos e às bases Literatura LatinoAmericana e do Caribe em Ciência Sociais (LILACS) e Base de Dados de Enfermagem (BDENF). Nas bases Health Systems Evidence (https://www.healthsystemsevidence.org/), Health Evidence (https://www.healthevidence.org/) e Epistemonikos (https://www.epistemonikos.org/), utilizou-se o termo "childbirth". Os detalhes das buscas estão disponíveis no Material Suplementar (página 5).

\section{Seleção e extração de dados}

A seleção foi realizada por duas revisoras (CFO, AAVR) na plataforma Rayyan QCRI (9). As divergências foram resolvidas por consenso. A extração foi feita por duas pesquisadoras (CFO, AAVR) de forma independente e revisada por outra dupla (MCB, TST), usando planilha eletrônica para registro das informações do estudo e das barreiras identificadas segundo nível de organização de sistemas de saúde (7).

\section{Avaliação da qualidade metodológica}

As revisões sistemáticas foram avaliadas em duplicidade com a ferramenta AMSTAR I (10), sendo classificadas em qualidade baixa (escore 0-3), moderada (4-7) ou alta (8-11). As divergências foram resolvidas por consenso. Quando disponível nas bases Health Evidence e Health Systems Evidence, foi utilizada a avaliação prévia do AMSTAR I. A qualidade metodológica das demais revisões não sistemáticas e dos guias de prática clínica não foi avaliada.

\section{Síntese dos achados}

As barreiras identificadas foram agrupadas segundo as orientações metodológicas da análise temática (11). Primeiramente, os relatos foram categorizados conforme significados comuns, considerando as oito seções das Diretrizes e os níveis organizacionais (usuários, trabalhadores, serviços e sistemas de saúde) (7). Em seguida, as categorias foram novamente analisadas e reagrupadas em núcleos temáticos por similaridade.

\section{RESULTADOS}

As buscas recuperaram 1611 documentos, que foram somados a outros 13 artigos e dois guias de práticas clínicas sugeridos por especialistas. No processo de seleção, 42 estudos foram excluídos (com justificativas), resultando na inclusão de 23 estudos na síntese de achados (figura 1).

\section{Figura 1. Fluxograma de seleção de estudos}

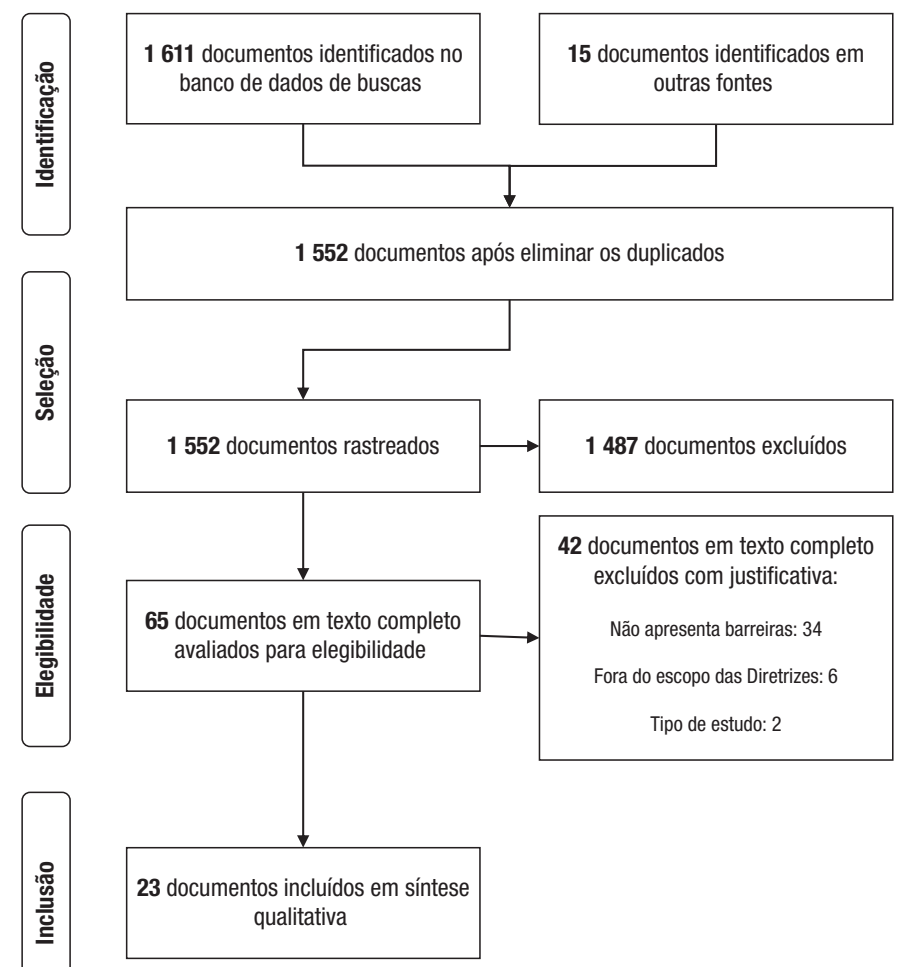




\section{Características dos estudos incluídos}

Incluíram-se 10 revisões sistemáticas, cinco sínteses de evidências qualitativas, quatro revisões narrativas, dois mapas de evidências e dois guias de prática clínica, constituídos por estudos primários de países de renda alta $(36,1 \%)$, média baixa $(31,8 \%)$, média alta $(20,3 \%)$ e baixa $(11,8 \%)$. Em relação às revisões sistemáticas, sete apresentaram qualidade moderada, duas apresentaram qualidade alta e uma apresentou qualidade baixa (Material Suplementar, página 9).

Os estudos incluídos abordaram barreiras relacionadas a cinco das oito seções das Diretrizes: 1) local de assistência ao parto, 2) cuidados gerais durante o trabalho de parto, 3) alívio da dor no trabalho de parto, 4) assistência no primeiro período do parto e 5) assistência no segundo período do parto. Os resultados se relacionaram a 25 das 225 recomendações das Diretrizes (Material Suplementar, página 15). A síntese dos achados, agrupada em nove núcleos temáticos e 52 categorias de barreiras, é descrita na tabela 1. Informações completas estão disponíveis no Material Suplementar (página 18).

\section{Modelo de atenção ao parto e nascimento}

O núcleo temático com mais categorias abordou o modelo de atenção ao parto e ao nascimento, com desafios relacionados aos serviços e sistemas de saúde. O modelo biomédico, hegemônico na assistência ao parto, molda e centra as práticas assistenciais no ambiente hospitalar, privilegiando a atuação do médico em detrimento dos demais profissionais de saúde $(12,13)$. Essa assimetria, influenciada por questões de gênero, dificulta a atuação de obstetrizes e enfermeiras obstetras. Nesse ambiente, a adoção de diferentes modelos de cuidado entre os profissionais gera conflitos ou interferências no cuidado oferecido (12-15). Além disso, a posição privilegiada de poder dos profissionais na relação com as usuárias interfere no cuidado prestado e na autonomia das mulheres (14-17), e a falta de vínculo entre profissionais de saúde e gestantes tem impacto na escolha da via de parto $(18,19)$.

A forma de organização dos sistemas de saúde, a legitimação de relações hierarquizadas e a falta de recursos financeiros, humanos, materiais e gerenciais, juntamente à baixa disponibilidade de políticas voltadas à promoção do cuidado respeitoso, são elementos que podem contribuir para a naturalização e a legitimação dos maus-tratos às mulheres durante o parto e o nascimento. Entre esses maus-tratos, incluem-se abusos físico, sexual e verbal; estigma e discriminação; e a adoção de padrões de cuidado inadequados em todo o mundo $(12,16,19,20)$.

As dificuldades na adoção de práticas baseadas em evidências e de um novo modelo de atenção ao parto estão relacionadas em parte à ausência de protocolos locais que amparem e explicitem essas ações $(14,21)$. Por outro lado, a imposição de protocolos e/ou normas rígidas também pode dificultar a oferta de um cuidado centrado na mulher $(12,13,17,21,22)$. Por exemplo, pode-se exigir das parteiras o atendimento das necessidades do hospital em detrimento das necessidades da usuária. Em contrapartida, o uso de protocolos também pode ser visto por algumas parteiras como fonte de pressão e interferência no cuidado prestado. A dificuldade em estabelecer medidas institucionais de apoio a práticas baseadas na fisiologia do nascimento, no respeito às usuárias e no cuidado centrado nas mulheres faz com que obstetrizes e enfermeiras obstetras vivenciem situações de assédio moral, estresse e síndrome de burnout $(12,13,17,22)$.

No ambiente hospitalar, valoriza-se o uso de tecnologias, a condução ativa do parto e as competências puramente técnicas $(12,13)$. Em muitos locais, as mulheres são forçadas a permanecerem deitadas durante o parto, seja por questões culturais ou por incapacidade dos profissionais para atender o parto em outras posições $(16,21,23)$.

\section{Gestão de recursos humanos}

As dificuldades na gestão de recursos humanos estão relacionadas aos níveis organizacionais dos trabalhadores e dos serviços e sistema de saúde. Há relatos de sobrecarga de trabalho dos profissionais, com jornadas extensas, sem intervalos e com muitos atendimentos $(12,17,20-22,24-26)$. O número reduzido de profissionais dificulta o cuidado adequado $(13,16$, $17,20,21,27)$. Essas barreiras são agravadas por rotinas hospitalares inadequadas que pressionam para um atendimento mais rápido, afetando a percepção das mulheres sobre a necessidade do alívio da dor e suas escolhas quanto às tecnologias $(16,19-22)$.

A falta de qualificação dos profissionais $(14-16,18,20,21)$ e a baixa remuneração $(13,15,16,20)$ podem ser barreiras ao acesso e à incorporação de novas práticas. O despreparo dos profissionais está relacionado à baixa compreensão dos direitos das usuárias $(17,18,22)$ e à baixa competência para receber os acompanhantes de modo a incorporá-los a todo o processo $(16,21,28)$. No contexto do local de trabalho, pode-se exigir a adoção de precauções de segurança focadas nas condições mínimas para os profissionais em detrimento das mulheres $(13,16)$.

As barreiras do nível organizacional do sistema de saúde estão relacionadas à formação dos profissionais. A exclusão da humanização como tema nos currículos acadêmicos $(14,22)$ e a falta de investimentos na formação e na permanência de obstetrizes e enfermeiras obstetras nos serviços (13-15) podem fomentar a continuidade de práticas inadequadas.

\section{Crenças e saberes}

Crenças e saberes de profissionais e de usuários podem representar barreiras que impactam o cuidado ofertado ao parto e nascimento. Algumas mulheres não desejam receber intervenções médicas durante o parto, o que pode interferir na escolha do local do parto; por outro lado, outras esperam intervenções, o que pode ser entendido pelos profissionais como indicador de sua passividade e aceitação do modelo de cuidado medicalizado $(12,16)$. As mulheres podem apresentar dificuldades em aceitar a analgesia epidural por medo do procedimento e de seus riscos (21); entretanto, a escolha da analgesia epidural como primeiro método de alívio da dor pode restringir o uso de métodos não farmacológicos (29).

Em contextos nos quais o ato sexual é visto como pecaminoso, os profissionais de saúde podem ter a percepção de que os maus-tratos e a vivência da dor durante o parto são punições adequadas para as gestantes $(15,21,30)$. Visões divergentes sobre o parto normal tornam aceitável, para alguns profissionais, o uso de tecnologias e, para outros, apenas o acompanhamento e apoio (12). Em países onde a episiotomia é praticada livremente, 
TABELA 1. Síntese dos achados sobre barreiras à implementação das recomendações por núcleo temático, nível de organização, categoria e seção das Diretrizes

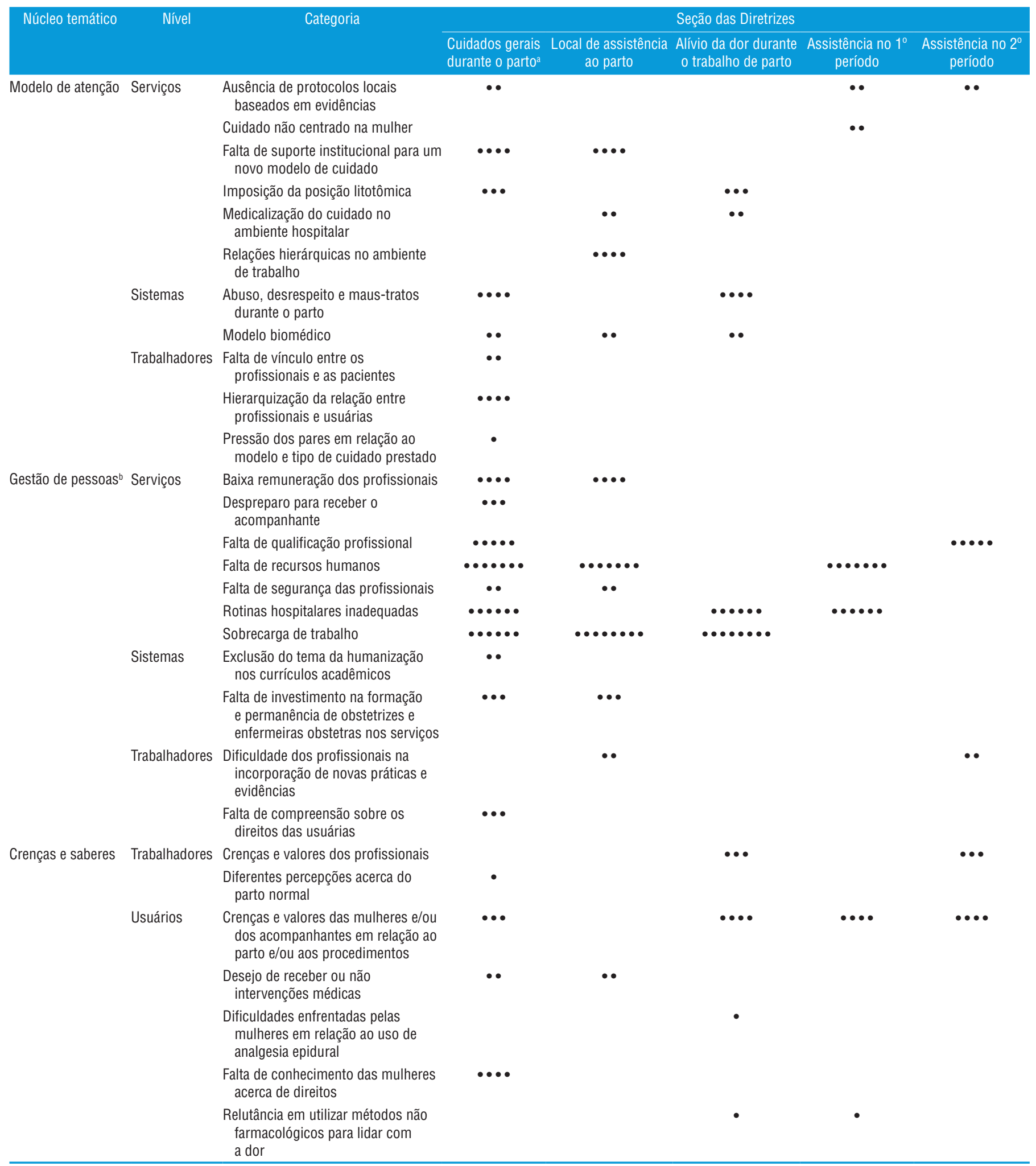


TABELA 1. Síntese dos achados sobre barreiras à implementação das recomendações por núcleo temático, nível de organização, categoria e seção das Diretrizes (continuação)

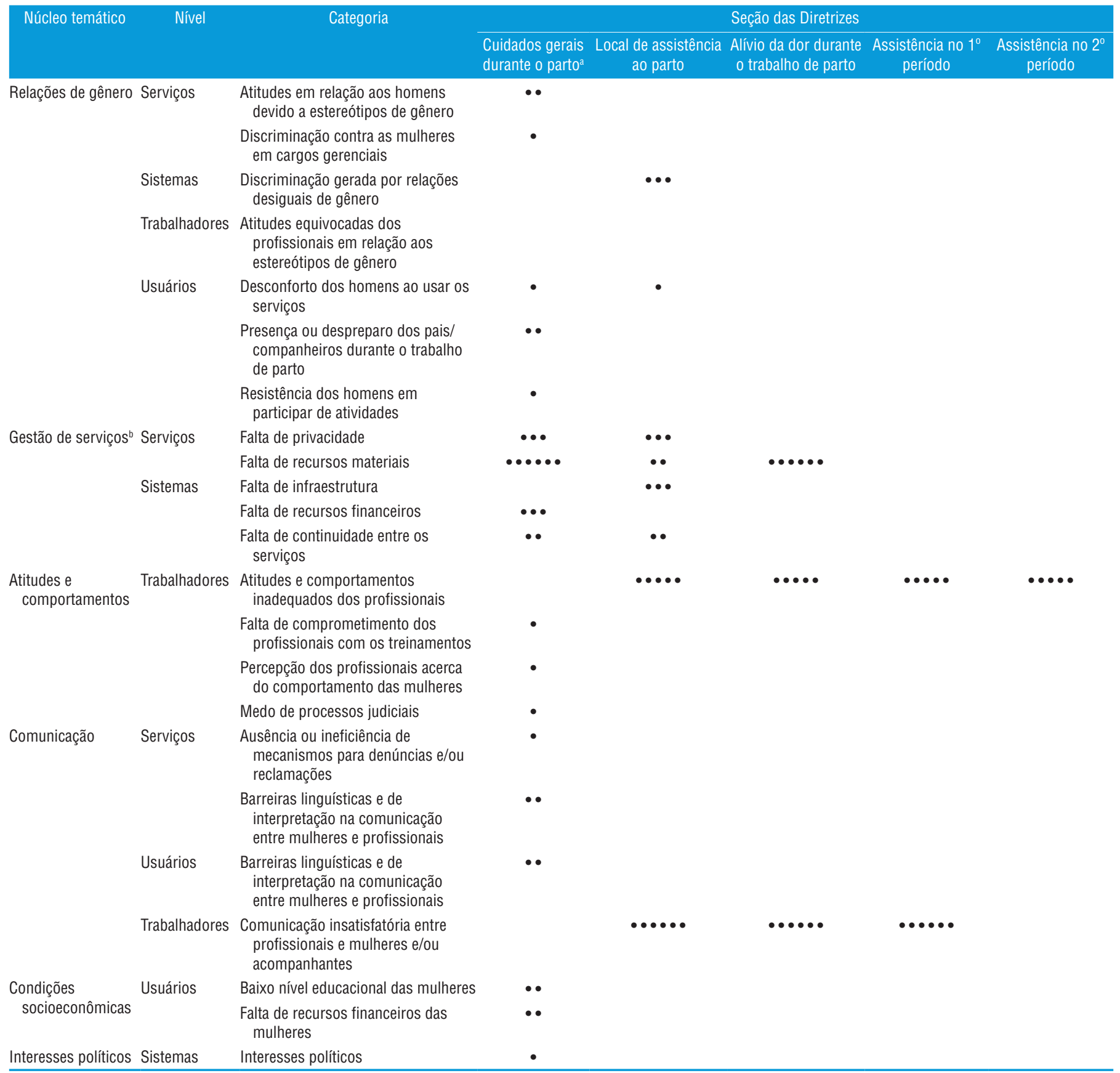

${ }^{a}$ Número de estudos relatando a barreira específica.

' Gestão de pessoas: recursos humanos; gestão de serviços: serviços de saúde.

pode haver a crença de que seu uso facilita o parto $(21,25,31$, 32). A falta de conhecimento das mulheres sobre seus direitos também interfere no acesso e no tipo de cuidado que recebem, além de na escolha do tipo de parto $(15,16,18,20)$.

\section{Relações de gênero}

Nesse núcleo temático, foram encontradas barreiras em todos os níveis de organização, relacionadas às atitudes dos homens e/ou de profissionais de saúde. De acordo com os estudos analisados, homens podem estar despreparados para lidar com o parto e ter a sensação de impotência e desamparo em relação ao sofrimento e à dor vivenciados pela parceira. Alguns apresentam resistência em participar de atividades de conscientização acerca dos direitos das mulheres (33). Os parceiros, em geral, não se sentem confortáveis utilizando os serviços de saúde, mesmo quando estão apenas acompanhando as mulheres (12). Apesar de as políticas de saúde de alguns países, como o Brasil, 
incentivarem a participação dos pais durante a gestação, o parto e o pós-parto, há serviços de saúde que ainda encontram dificuldades para inserir esses homens no processo $(31,32)$. Para algumas mulheres, a presença do companheiro durante o parto também pode ser um fator estressante $(25,28)$.

O comportamento dos profissionais em relação aos estereótipos de gênero afeta o tipo de cuidado oferecido às mulheres e aos acompanhantes. Mulheres que não se conformam às normas e aos estereótipos de feminilidade podem sofrer maus-tratos, e mulheres em cargos gerenciais tendem a ser mais discriminadas (15). A discriminação, por parte dos médicos, em relação a obstetrizes e enfermeiras obstetras, bem como a falta de incentivo à formação e atuação autônoma dessas profissionais, são fatores influenciados por relações desiguais de gênero. As profissionais mulheres vivenciam maior desgaste profissional, além de receberem salários menores. O cuidado, por ser uma atividade atribuída às mulheres, é menos valorizado. Com relação às usuárias, sua falta de autonomia durante o parto e as violências sofridas durante o período gravídico-puerperal também estão relacionadas a questões culturais ligadas às relações de gênero $(13,15,22)$.

\section{Gestão de serviços de saúde}

Na dimensão da gestão dos serviços e sistemas de saúde, a falta de medicamentos e outros insumos é uma barreira para a assistência adequada ao parto $(13,16,17,20,21,29)$. Problemas de infraestrutura podem prejudicar a privacidade da mulher, e a ausência de espaços adequados impede a presença de acompanhantes $(16,19,21)$.

Questões de infraestrutura, como saneamento básico e transporte, podem dificultar o acesso aos serviços (13, 21, 32). No âmbito da gestão do sistema, as dificuldades podem estar relacionadas à falta de repasses financeiros, com impacto sobre a contratação e a manutenção de profissionais e a melhoria da infraestrutura $(16,20,21)$. Dificuldades na articulação e no referenciamento entre os serviços de saúde também impedem a continuidade do cuidado $(18,19)$.

\section{Atitudes e comportamentos}

As atitudes e os comportamentos dos profissionais em relação às mulheres, como condutas impróprias, negligência e falta de compaixão, podem interferir no cuidado prestado e nas escolhas das mulheres $(16,20,21,23,26)$. Ademais, a falta de comprometimento dos profissionais com os treinamentos ofertados (24) e o medo de sofrerem processos judiciais influenciam suas práticas (12).

\section{Comunicação}

As barreiras na comunicação entre profissionais, usuárias e seus acompanhantes podem fazer com que as mulheres não obtenham as informações adequadas ou necessárias para seu parto $(16,18,20,21,24,34)$. A ausência de intérpretes (por exemplo, na presença de pacientes de outras nacionalidades) e o impedimento da presença de familiares e acompanhantes nas unidades de saúde reforçam as barreiras de comunicação $(16,19)$. Além disso, a ausência ou ineficiência dos mecanismos de reclamações ou denúncia faz com que situações de violência não sejam adequadamente apuradas (16).

\section{Condições socioeconômicas}

O baixo nível educacional e a escassez de recursos financeiros das mulheres podem interferir negativamente no acesso e no tipo de cuidado que recebem $(15,20)$.

\section{Interesses políticos}

Uma dificuldade enfrentada pelo sistema de saúde é a cooptação dos interesses de organizações não governamentais pelo governo quando são incorporadas a iniciativas governamentais. Além disso, disputas partidárias nas comunidades podem afetar a conscientização de meninas e mulheres acerca de seus direitos (17).

\section{DISCUSSÃO}

No contexto brasileiro, a identificação de barreiras para adesão às recomendações das Diretrizes é um passo importante no processo de tradução do conhecimento (7). Os achados revelam a necessidade de os tomadores de decisão e demais atores sociais envolvidos considerarem estratégias de mudança do modelo tecnocrático vigente, focando nas dificuldades de comunicação entre usuários e profissionais, no gerenciamento dos recursos humanos e dos serviços de saúde, nas questões econômicas e socioculturais, nas violências de gênero e nos interesses políticos que impedem a ampla adoção das boas práticas.

Dificuldades relatadas pelas mulheres na articulação dos seus desejos em relação à gestação e ao parto com os profissionais de saúde, bem como no entendimento das informações passadas por eles, estiveram relacionadas ao baixo grau de escolaridade das usuárias. A baixa escolaridade e o baixo nível socioeconômico maternos estão relacionados a menor adesão ao cuidado pré-natal e participação em ações educativas (35, 36). Essa realidade faz com que as mulheres desenvolvam pouco conhecimento sobre a fisiologia do trabalho de parto e sobre seus direitos, o que interfere nas expectativas geradas por elas em relação ao processo de nascimento e percepção sobre a assistência recebida, uma importante barreira na luta contra a violência obstétrica $(37,38)$.

Barreiras na comunicação entre mulheres e profissionais guardam relação também com o atual modelo de atenção à saúde, no qual o diálogo entre as mulheres, as famílias, os profissionais e os gestores é praticamente inexistente (1). É necessário considerar a influência de questões de gênero na atenção obstétrica, expressas na dificuldade de inserção dos homens nas discussões sobre a gestação e no cenário do parto, nos abusos e maus-tratos às gestantes, na discriminação contra mulheres que exercem cargos gerenciais e na falta de incentivo para a formação, contratação e manutenção de obstetrizes e enfermeiras obstetras na atenção ao pré-natal, parto e puerpério.

Resultados preliminares da Rede Cegonha (39) e do projeto Parto Adequado mostram aumento de $31,8 \%$ para $83,9 \%$ na presença de acompanhante no parto vaginal no setor público e de $55,8 \%$ para $96,8 \%$ no privado. Entretanto, com base nos dados, não é possível dizer se os acompanhantes foram a primeira escolha dessas mulheres, nem tampouco como os acompanhantes foram recebidos nas unidades. Estudos qualitativos têm mostrado que os homens enfrentam dificuldades nesses locais, sem permissão para permanecer o tempo todo com a mulher, além de serem impedidos de participar ativamente no apoio $(40,41)$. 
Entretanto, há experiências que mostram estímulo à participação do pai no momento do nascimento e o quanto essas ações são importantes para a promoção da humanização nos serviços e para a desconstrução de uma masculinidade que é negativa para o indivíduo e para suas relações com a parceira, os filhos e a sociedade $(42,43)$. É necessário, portanto, pensar estratégias que consigam garantir o direito de acompanhante a todas as mulheres, como a sensibilização dos profissionais e usuários, a alocação de recursos financeiros e o comprometimento dos gestores (44), uma vez que a presença de um acompanhante está relacionado ao aumento da satisfação da mulher com o processo de nascimento e da adoção das boas práticas assistenciais por parte dos profissionais $(41,45)$.

Os abusos e desrespeitos contra mulheres no contexto do nascimento são reconhecidos pela Organização Mundial da Saúde (OMS) como um problema global de saúde pública, que se alicerçam em estereótipos de gênero presentes na formação dos profissionais de saúde e na organização dos serviços (46). Dentro dessa ótica, o corpo da mulher é visto como imperfeito e também como um fator de risco, demandando ajustes e tutela, que culminam na desqualificação da sexualidade das mulheres, com atitudes autoritárias, discriminatórias e iatrogênicas pelos profissionais (11).

A dificuldade na inserção e manutenção de outros atores na assistência ao parto, muito influenciada por questões de gênero, também é uma realidade brasileira. No país, o número de enfermeiras obstetras é desconhecido; a percepção, porém, é de que sejam poucas e, muitas vezes, alocadas para outros setores que não o obstétrico (47). Em 2008, apenas $8 \%$ dos partos normais foram atendidos por enfermeiras obstetras (48). Após os incentivos da Rede Cegonha, esse percentual aumentou para $27 \%$ no setor público, sendo praticamente inexistente no setor privado (1,8\% em 2017) (39). Com o intuito de propiciar a formação de profissionais especialistas em nascimentos fisiológicos desde a graduação e aumentar a oferta de parteiras nos serviços de saúde, a Universidade de São Paulo (USP) voltou a oferecer, em 2005, o curso de graduação em Obstetrícia (49). As obstetrizes, porém, enfrentam desafios para sua completa inserção e atuação nos serviços de saúde, mesmo que as experiências mostrem impacto positivo do seu exercício na atenção ao parto e nascimento $(47,50)$.

O número reduzido de trabalhadoras na assistência tem como consequência o aumento na sobrecarga de trabalho, somado aos baixos salários e às rotinas inadequadas (50-52). Outro ponto a ser destacado refere-se às fragilidades no processo de formação e atualização dos profissionais. Um estudo qualitativo que avaliou barreiras e facilitadores em hospitais da América Latina mostrou que a falta de disposição dos profissionais em mudar suas práticas foi determinada na universidade, pois não foram capacitados para perceber que, na Medicina, o conhecimento é dinâmico, e não desenvolveram habilidades suficientes para entendimento da literatura científica (53). Há necessidade de inclusão de discussões sobre a humanização do nascimento, gênero e direitos das mulheres na formação dos profissionais de saúde para a qualificação efetiva das práticas obstétricas $(46,54)$.

Por fim, o enfrentamento das barreiras identificadas não será possível sem a gestão adequada dos serviços de saúde. Problemas de infraestrutura nos serviços, número insuficiente de profissionais, saneamento básico e transporte insuficientes e falta de recursos ou de repasses financeiros também devem ser considerados.

\section{Limitações do estudo}

Deve-se considerar que esta revisão rápida adotou atalhos metodológicos previamente definidos e considerados cuidadosamente pelos autores, tais como a limitação das buscas e a seleção e extração dos artigos por um único revisor. Algumas limitações importantes devem ser consideradas na interpretação dos resultados. Primeiro, a decisão de limitar as bases revisadas se deu em face do limite temporal para a realização do estudo. Assim, os impactos da abrangência desta revisão devem ser considerados na interpretação dos resultados apresentados. Segundo, foram incluídos apenas estudos secundários e guias de prática clínica, o que pode ter contribuído para a não identificação de barreiras para várias recomendações das Diretrizes. As revisões narrativas têm nível de confiança de evidência mais baixo, mas abordam barreiras que parecem relevantes para o contexto do Brasil, visto que todas são produções nacionais. Terceiro, apenas as revisões sistemáticas tiveram sua qualidade avaliada e, embora representem a parte importante dos estudos incluídos, deve-se ter cautela e considerar as eventuais incertezas sobre a confiabilidade dos resultados apresentados pelos outros tipos de estudos incluídos, como as revisões narrativas. Por fim, a ausência de barreiras relacionadas à assistência no terceiro período do parto, aos cuidados maternos imediatamente após o parto e à assistência ao recém-nascido pode indicar que seria necessário incluir outros termos de busca mais específicos para esses períodos da assistência.

\section{Considerações finais}

Os achados desta revisão rápida apontam um problema que não é novo, tampouco desconhecido. Visualizar explicitamente barreiras à implementação das Diretrizes enseja que estratégias fundamentadas, bem delineadas e pactuadas com os atores envolvidos sejam adotadas para superá-las. Vislumbra-se a complexidade da atenção ao parto, principalmente com vistas à promoção do parto natural, que, por muitos anos, vem sendo desafiada pela medicalização da assistência obstétrica no mundo, inclusive no Brasil.

A superação das barreiras identificadas para implementação pode favorecer as boas práticas assistenciais e reduzir as altas taxas cesarianas desnecessárias e de mortes maternas e neonatais evitáveis, além de recuperar o potencial transformador da experiência de nascimento para a mulher, a criança e a sociedade. Ações conjuntas podem ser necessárias para promover a melhoria das condições financeiras e educacionais das mulheres e famílias, melhorar os mecanismos de gestão dos equipamentos e profissionais de saúde e conscientizar e combater as iniquidades de gênero e as violências a elas atreladas.

A implementação efetiva das Diretrizes e a construção de um modelo centrado nas necessidades de cada mulher e família necessitarão do comprometimento de gestores e profissionais com as boas práticas assistenciais, além da garantia da continuidade das estratégias e políticas de saúde em andamento, como a Rede Cegonha, a partir de ações de incentivo e monitoramento. Ademais, as barreiras socioeconômicas encontradas demandam uma sociedade mais justa e acolhedora, com promoção da autonomia e do respeito aos indivíduos, a começar pelo momento do nascimento. 
Contribuição dos autores. JOMB e EMGC conceberam o estudo. CFO, AAVR e MCB realizaram busca, extração, síntese e interpretação dos dados e redigiram a primeira versão do manuscrito. CDLJ participou da interpretação dos dados. TST participou da busca e interpretação dos dados. Todos os autores contribuíram, revisaram e aprovaram a versão final do manuscrito.

Conflitos de interesse. JOMB recebe salário da Fundação Oswaldo Cruz (Fiocruz), TST e MCB recebem salário do Instituto de Saúde de São Paulo. CFO, AAVR e CDLJ receberam bolsa de pesquisa. Os autores declaram que não possuem conflitos de interesses que tenham afetado a condução do estudo ou a apresentação dos resultados.

Financiamento. Este estudo foi financiado pela Organização Pan-Americana da Saúde (OPAS), pelo Programa Especial de Pesquisa e Treinamento em Doenças Tropicais (TDR) e pela Aliança para Pesquisa em Políticas e Sistemas de Saúde (AHPSR) através de uma doação ao projeto "Barreiras e estratégias para a implementação das Diretrizes do Parto Normal no Brasil [Chamada para propostas "Incorporação de pesquisas para os Objetivos de Desenvolvimento Sustentável (ER-SDG)]". Os financiadores não tiveram nenhum papel no desenho, análise, interpretação dos dados e redação do manuscrito.

Agradecimentos. Os autores agradecem a Taís R. Tesser pela assistência prestada nas etapas preparatórias desta revisão, bem como aos participantes do Diálogo Deliberativo, realizado na Fiocruz Brasília em junho de 2019, pelo feedback sobre os achados preliminares do estudo.

Declaração. As opiniões expressas no manuscrito são de responsabilidade exclusiva dos autores e não refletem necessariamente a opinião ou política da RPSP/PAJPH ou da Organização Pan-Americana da Saúde (OPAS).

\section{REFERÊNCIAS}

1. Pasche DF, Vilela ME de A, Giovanni M Di, Almeida PVB, Franco Netto T de L. Rede Cegonha: desafios de mudanças culturais nas práticas obstétricas e neonatais. Divulg saúde debate. 2014;52:58-71.

2. Leal MC, Silva AAM, Dias MAB, Gama SGN, Rattner D, Moreira $\mathrm{ME}$, et al. Birth in Brazil: national survey into labour and birth. Reprod Health. 2012;9:15

3. Boerma T, Ronsmans C, Melesse DY, Barros AJD, Barros FC, Juan $\mathrm{L}$, et al. Global epidemiology of use of and disparities in caesarean sections. Lancet. 2018;392(10155):1341-8.

4. Brasil. Ministério da Saúde. Diretrizes nacionais de assistência ao parto normal: versão resumida [Internet]. Brasília: Ministério da Saúde; 2017 [citado 2020 jun 2]. Disponível em: https://pesquisa. bvsalud.org/bvsms/resource/pt/mis-39026

5. Latosinsky S, Fradette K, Lix L, Hildebrand K, Turner D. Canadian breast cancer guidelines: have they made a difference? CMAJ. 2007;176(6):771-6.

6. Gagliardi AR, Brouwers MC, Palda VA, Lemieux-Charles L, Grimshaw JM. How can we improve guideline use? A conceptual framework of implementability. Implement Sci. 2011;6(1):26.

7. Fretheim A, Munabi-Babigumira S, Oxman AD, Lavis JN, Lewin S. SUPPORT Tools for Evidence-informed Policymaking in health 6: Using research evidence to address how an option will be implemented. Heal Res Policy Syst. 2009;7(S1):S6.

8. Tricco AC, Langlois E V, Straus SE (eds). Rapid reviews to strengthen health policy and systems: a practical guide. Geneva: World Health Organization; 2017.

9. Ouzzani M, Hammady H, Fedorowicz Z, Elmagarmid A. Rayyan-a web and mobile app for systematic reviews. Syst Rev. 2016;5:210

10. Shea BJ, Grimshaw JM, Wells GA, Boers M, Andersson N, Hamel C, et al. Development of AMSTAR: a measurement tool to assess the methodological quality of systematic reviews. BMC Med Res Methodol. 2007;7(1):10.

11. Minayo MCS (ed.), Deslandes SF, Gomes R. Pesquisa social: teoria, método e criatividade. Rio de Janeiro, Brasil: Vozes; 2013.

12. O'Connell R, Downe S. A metasynthesis of midwives' experience of hospital practice in publicly funded settings: compliance, resistance and authenticity. Health (Irvine Calif). 2009;13(6):589-609.

13. Filby A, McConville F, Portela AG. What Prevents Quality Midwifery Care? A Systematic Mapping of Barriers in Low and Middle Income Countries from the Provider Perspective. PLoS One. 2016;11(5):e0153391.

14. Gomes ML, Moura MAV. Modelo humanizado de atenção ao parto no Brasil: evidências na produção científica. Rev Enferm UERJ. 2012;20(2):248-53.
15. Betron ML, McClair TL, Currie S, Banerjee J. Expanding the agenda for addressing mistreatment in maternity care: a mapping review and gender analysis. Reprod Health. 2018;15(1):143.

16. Bohren MA, Vogel JP, Hunter EC, Lutsiv O, Makh SK, Souza JP, et al. The Mistreatment of Women during Childbirth in Health Facilities Globally: A Mixed-Methods Systematic Review. PLoS Med. 2015;12(6):e1001847.

17. George AS, Branchini C. Principles and processes behind promoting awareness of rights for quality maternal care services: a synthesis of stakeholder experiences and implementation factors. BMC Pregnancy Childbirth. 2017;17(1):264.

18. Avanci BS, Cortez EA, Barbosa FS, André KM. Papel do enfermeiro na perspectiva do Programa de Humanização do pré-natal, parto natural e nascimento: revisão sistemática de literatura. Rev Enferm UFPE Line. 2009;3(4):1126-33.

19. Jones E, Lattof SR, Coast E. Interventions to provide culturally-appropriate maternity care services: factors affecting implementation. BMC Pregnancy Childbirth. 2017;17(1):267.

20. Ishola F, Owolabi O, Filippi V. Disrespect and abuse of women during childbirth in Nigeria: A systematic review. PLoS One. 2017;12(3):e0174084.

21. World Health Organization. WHO recommendations: intrapartum care for a positive childbirth experience. Geneva: World Health Organization; 2018.

22. Shakibazadeh E, Namadian M, Bohren M, Vogel J, Rashidian A, Nogueira Pileggi V, et al. Respectful care during childbirth in health facilities globally: a qualitative evidence synthesis. BJOG An Int J Obstet Gynaecol. 2018;125(8):932-42.

23. Vargens OMC, Silva ACV, Progianti JM. Non-invasive nursing technologies for pain relief during childbirth-The Brazilian nurse midwives' view. Midwifery. 2013;29(11):e99-106.

24. Chang Y-S, Coxon K, Portela AG, Furuta M, Bick D. Interventions to support effective communication between maternity care staff and women in labour: A mixed-methods systematic review. Midwifery. 2018;59:4-16.

25. Lunda P, Minnie CS, Benadé P. Women's experiences of continuous support during childbirth: a meta-synthesis. BMC Pregnancy Childbirth 2018;18(1):167.

26. Lyberg A, Dahl B, Haruna M, Takegata M, Severinsson E. Links between patient safety and fear of childbirth-A meta-study of qualitative research. Nurs Open. 2019;6(1):18-29.

27. Long Q, Allanson ER, Pontre J, Tunçalp Ö, Hofmeyr GJ, Gülmezoglu AM. Onsite midwife-led birth units (OMBUs) for care around the time of childbirth: a systematic review. BMJ Glob Health. 2016;1(2):e000096. 
28. Hoga LAK, Reberte Gouveia LM, Higashi AB, de Souza Zamo-Roth F. The experience and role of a companion during normal labor and childbirth: a systematic review of qualitative evidence. JBI Database Syst Rev Implement Reports. 2013;11(12):121-56.

29. Shaw-Battista J. Systematic Review of Hydrotherapy Research. J Perinat Neonatal Nurs. 2017;31(4):303-16.

30. Renfrew MJ, Hannah W, Albers L, Floyd E. Practices That Minimize Trauma to the Genital Tract in Childbirth: A Systematic Review of the Literature. Birth. 1998;25(3):143-60.

31. Mazzieri SPM, Hoga LAK. Participação do pai no nascimento e parto: revisão da literatura. Rev Min Enferm. 2006;10(3):166-70.

32. Miltenburg AS, Roggeveen Y, van Roosmalen J, Smith H. Factors influencing implementation of interventions to promote birth preparedness and complication readiness. BMC Pregnancy Childbirth. 2017;17(1):270

33. George AS, Branchini C, Portela AG. Do Interventions that Promote Awareness of Rights Increase Use of Maternity Care Services? A Systematic Review. PLoS One. 2015;10(10):e0138116.

34. World Health Organization. WHO recommendations: non-clinical interventions to reduce unnecessary caesarean sections. Geneva: World Health Organization; 2018.

35. Domingues RMSM, Hartz ZMA, Dias ABM, Leal MC. Avaliação da adequação da assistência pré-natal na rede SUS do Município do Rio de Janeiro, Brasil. Cad Saude Publica. 2012;28(3):425-37.

36. Costa CSC, Vila VDC, Rodrigues FM, Martins CA, Pinho LMO. Características do atendimento pré-natal na Rede Básica de Saúde. Rev Eletrônica Enferm. 2013;15(2):516-22.

37. Hotimsky SN, Rattner D, Venancio SI, Bógus CM, Miranda MM. O parto como eu vejo... ou como eu o desejo?: expectativas de gestantes, usuárias do SUS, acerca do parto e da assistência obstétrica. Cad Saude Publica. 2002;18(5):1303-11.

38. Reis CC, Souza KRF, Alves DS, Tenório IM, Brandão W. Percepção das mulheres sobre a experiência do primeiro parto: implicações para o cuidado de enfermagem. Cienc y Enferm. 2017;XXIII(2):45-56.

39. Leal MC, Bittencourt SA, Esteves-Pereira AP, Ayres BVS, Silva LBRAA, Thomaz EBAF, et al. Avanços na assistência ao parto no Brasil: resultados preliminares de dois estudos avaliativos. Cad Saude Publica. 2019;35(7):e00223018.

40. Souza SRRK, Gualda DMR. A experiência da mulher e de seu acompanhante no parto em uma maternidade pública. Texto Contexto Enferm. 2016;25(1):e4080014.

41. Bohren MA, Berger BO, Munthe-Kaas H, Tunçalp Ö. Perceptions and experiences of labour companionship: A qualitative evidence synthesis. Cochrane Database Syst Rev. 2019;3:CD012449.

42. Braide ASG, Brilhante AV, Arruda CND, Mendonça FADC, Caldas JMP, Nations MK, Amorin RFD. Sou homem e pai sim!(Re) construindo a identidade masculina a partir da participação no parto. Rev Panam Salud Publica. 2018;42:e190). https:/ / doi.org/10.26633/ RPSP.2018.190
43. Matos MG, Magalhães AS, Féres-Carneiro T, Machado RN. Construindo o Vínculo Pai-Bebê: A Experiência dos Pais. Psico-USF 2017;22(2):261-271.

44. Kabakian-Khasholian T, Portela A. Companion of choice at birth: factors affecting implementation. BMC Pregnancy Childbirth. 2017;17(265):1-13

45. Monguilhott JJDC, Brüggemann OM, Freitas PF, d'Orsi E. Nascer no Brasil: a presença do acompanhante favorece a aplicação das boas práticas na atenção ao parto na região Sul. Revista Saúde Pública. 2018;52(1):1-11.

46. Diniz CSG, Salgado HO, Andrezzo HFA, de Carvalho PGC, Carvalho PCA, Aguiar C, et al. Violência obstétrica como questão para a saúde pública no Brasil: origens, definições, tipologia, impactos sobre a saúde materna, e propostas para sua prevenção. J Hum Growth Dev. 2015;25(3):377-376.

47. Narchi NZ, de Castro CM, Oliveira C de F, Tambellini F. Report on the midwives' experiences in the Brazilian National Health System: A qualitative research. Midwifery. 2017;53:96-102.

48. Victora CG, Aquino EM, do Carmo Leal M, Monteiro CA, Barros FC, Szwarcwald CL. Maternal and child health in Brazil: progress and challenges. Lancet. 2011;377(9780):1863-76.

49. Narchi NZ, da Silva LCFP, Gualda DMR, Bastos MH. Reclaiming direct-entry midwifery training in Brazil: Context, challenges and perspectives. Midwifery. 2010;26(4):385-8.

50. Lima CF, Soares GCF, Gualda DMR. Midwives' Experiences: inconsistencies in education vs. clinical practice. Rev Iberoam Educ Invest Enferm. 2019;9(2):7-18.

51. Oliveira RJT, Copelli FHS, Pestana AL, Santos JLG, Gregório VRP, Erdmann AL. Intervening conditions on governance of the nursing practice at an obstetrics centre. Rev Gaúcha Enferm. 2014;35(1):47-54.

52. Progianti JM, Moreira NJM de P, Prata JA, Vieira MLC, Almeida TA, Vargens OM da C. Job insecurity among obstetric nurses. Rev Enferm UERJ. 2018;26:e33846.

53. Belizan M, Meier A, Althabe F, Codazzi A, Colomar M, Buekens P, et al. Facilitators and barriers to adoption of evidence-based perinatal care in Latin American hospitals: a qualitative study. Health Educ Res. 2007;22(6):839-53.

54. Morais LO, Potros FR, Maia PR, Rabelo KLMA, Ribeiro FM, Matos KLA de, et al. O Parto Humanizado no contexto atual: Uma revisão integrativa. Rev Eletrônica Acervo Saúde. 2019;37:e1375.

Manuscrito recebido em 11 de julho de 2020. Aceito em versão revisada em 18 de setembro de 2020 . 


\section{Barriers to implementing guideline recommendations to improve childbirth care: rapid review of evidence}

ABSTRACT Objective. To identify potential barriers to the implementation of the National Childbirth Guidelines in Brazil based on the best available global evidence.

Method. A rapid review of evidence was performed in six databases in March/April 2019. Secondary studies published in English, Spanish, or Portuguese with a focus on barriers of any nature relating to the implementation of the Guidelines were retrieved.

Results. Twenty-three documents (21 reviews and two practice guides) were included in the review. The barriers identified were grouped into 52 meaning categories and then reorganized into nine thematic clusters: delivery and childbirth care model, human resource management, knowledge and beliefs, gender relations, health care service management, attitudes and behaviors, communication, socioeconomic conditions, and political interests.

Conclusions. The results show that combined approaches may be required to address different barriers to the implementation of the Guidelines. For successful implementation, it is essential to engage health care leaders, professionals, and users in the effort to change the delivery and childbirth care model. Also necessary is the development of intersectoral initiatives to improve the socioeconomic conditions of women and families and to curtail gender inequalities.

Keywords $\quad$ Evidence-informed policy; implementation science; practice guidelines as topic; parturition; Brazil.

\section{Obstáculos a la aplicación de las recomendaciones para la atención del parto normal: revisión rápida de evidencia}

RESUMEN Objetivo. Identificar los posibles obstáculos a la aplicación de las recomendaciones formuladas en las Directrices Nacionales para la Atención del Parto Normal en Brasil a partir de la mejor evidencia disponible a nivel mundial.

Métodos. En marzo/abril de 2019 se llevó a cabo una revisión rápida de seis bases de datos. Se seleccionaron estudios secundarios publicados en español, inglés o portugués sobre los obstáculos de cualquier tipo que pudieran estar relacionados con la aplicación de las recomendaciones contenidas en las Directrices.

Resultados. Se incluyeron 23 documentos (21 revisiones sistemáticas y 2 guías de práctica clínica). Los obstáculos identificados se agruparon en 52 categorías con base en su semejanza de significado y luego se reorganizaron en nueve grupos temáticos: modelo de atención del parto, gestión de recursos humanos, creencias y conocimientos, relaciones de género, gestión de servicios de salud, actitudes y comportamientos, comunicación, condiciones socioeconómicas e intereses políticos.

Conclusiones. La aplicación de las Directrices puede requerir enfoques combinados para hacer frente a diferentes obstáculos. La participación de los administradores y los trabajadores de la salud en el proceso de cambio del modelo de atención del parto, así como la participación de los usuarios, son fundamentales para que la aplicación de las Directrices sea satisfactoria. Además, se necesitan medidas intersectoriales para mejorar las condiciones socioeconómicas de las mujeres y las familias y para combatir las desigualdades entre los géneros.

Palabras clave Política informada por la evidencia; ciencia de la implementación; guías de práctica clínica como asunto; parto; Brasil. 